\title{
Characterization of Distribution Pattern of Eye Fixation Pauses in Observation of Knotty Wood Panel Images
}

\author{
Masashi Nakamura and Takayuki Kondo
}

Kyoto University

\begin{abstract}
Wood is widely accepted as a human-friendly material. Many people use a large amount of wood in their living space. However, information on why and how humans are affected by wood is insubstantial. Understanding what a person is focusing on when looking at wood is an important first step in solving the above problem. The manner and style of our wood observation can be extracted directly by using an eye-tracker. The purpose of this study is to characterize the distribution pattern of eye fixation pauses in observing wood including many knots. Fifty-five kinds of wood panel images were prepared as visual stimuli. Various sizes of knots appeared on most of them. Twenty subjects observed these images for about 20 seconds per image freely with the eyetracker on their heads. Their eye movements were recorded as eyemarks during the observations, and many eye fixation pauses on each image were extracted for each subject. To express the distribution pattern of the eye fixation pauses quantitatively, two numerical indexes, the aspect ratio and the rate of expansion, were proposed in this study. The former index indicated the overall shape of the distribution, and the latter corresponded to the size of the observation area. Based on the relationships between these indexes, the distribution patterns were classified into three types. J Physiol Anthropol 26(2): 129-133, 2007 http://www.jstage.jst.go.jp/browse/jpa2
\end{abstract} [DOI: 10.2114/jpa2.26.129]

Keywords: eye movement, eyemark, eye fixation pause, eyetracker, wood, knot

\section{Introduction}

As for building material of a natural origin including wood, it is often pointed out that the affinity with humans is high. Even if one lives in a high-rise condominium which is a lump of iron and concrete, it is common to use a large amount of wood or wood-based materials for the interior, which is seen and touched directly. This might be a reflection of one's empirical knowledge about the affinity of wood. What is the significance of the moderate stimulation that the wooden environment gives for people who live in an urbanized modern society?

To make clear this problem, the authors have investigated the recognition characteristics of humans who observe various wood and wood-based materials. In our research process, we found the unexpected fact that many people blindly assume wood is good. Our evaluation regarding knots, in particular, highlighted great differences. If people are surrounded with knotty walls, one might feel discomfort, or another one might feel so good as if staying in a log cabin. Though knots are the remains of branches and the evidence that wood has grown as a living tree, the existence of knots causes the deterioration of the mechanical properties of lumber and is also undesirable in visual design. So lumber with few knots is traded at high prices in the market. Moreover, it might well correspond with the temperament of the Japanese; "the religion of being knotfree" has been nurtured in Japan for a long period. Recently the number of consumers who do not greatly mind the existence of knots has increased, and the interior with many knots is not novel today owing to the boom of natural building materials. But knots are a conspicuous feature of wood for humans. In our investigations (Masuda and Nakamura, 1987; Nakamura et al., 1993), though wall panels with knots evoked "natural" impressions very well, "agreeable" impressions declined as the amount of knots increased. Broman reported that many people in Northern Europe that had a culture of plain wood similar to Japan's also tended to prefer knot-free lumber to knotty lumber (Broman, 1995, 1996, 2001). Sakuragawa et al. (2005) carried out a sensory evaluation and the continuous measurement of blood pressure using the visual stimuli of a knotty hinoki-wood (Japanese cypress) board and a white painted steel board. In this experiment, visual stimulation from the hinoki-wood board also had an emotional and natural impression upon humans. Blood pressure decreased significantly in subjects who liked them. Interestingly, there was no significant increase in blood pressure in subjects who disliked the hinoki-wood board.

These studies suggest that knots of wood exert various influences upon humans psychologically and physiologically as a visual stimulus, and that the degree of this influence is 
different in each observer. In other words, although wood might be one of the indispensable interior materials for humans who live in an urbanized modern society, the influence of it on humans must be evaluated and classified from the point of view of physiological anthropology to demonstrate the optimum usage of wood for people. Understanding which part of wood a person is looking at is the first step to approaching this goal. The authors have tried to grasp the manner of our wood observation directly by using an eye-tracker. The purpose of this study is to characterize the distribution pattern of eye fixation pauses in observing wood including many knots.

\section{Methods}

\section{Subjects}

Twenty healthy Japanese students participated in this study. They were given sufficient information about the objective and procedure of the experiment. After this explanation, they subscribed to the agreement form.

\section{Visual stimuli}

A lot of sugi-wood laminae (Japanese cedar; $110 \mathrm{~mm}$ $(\mathrm{W}) \times 1000 \mathrm{~mm}(\mathrm{~L}) \times 12 \mathrm{~mm}(\mathrm{~T})$, from the Yoshino area of Nara Prefecture, Japan) were sorted, based on the size of knots. Photos were taken using a digital still camera. After the photoretouching process, these images were arranged on a computer screen. Finally, 55 kinds of wall panel images were generated. The minimum, mean, and maximum numbers of knots in the wall panel images were 28,61 , and 142 , respectively. The total areas were $1.3,43.1$, and $100.5 \mathrm{~cm}^{2}$, respectively. Many small pin-knots were included in the total area, as well as large knots. The amount of knots and the width and color of the seam between the laminae on each wall panel image were varied systematically. Fifty-five kinds of images were displayed on a large liquid crystal display (LCD monitor; SONY, KDX46Q005) in their actual size as visual stimuli for the experiment (three images as examples are shown in the upper row of Fig. 1). The displayed size of the images on the LCD monitor was about $950 \mathrm{~mm} \times 570 \mathrm{~mm}$; the luminance of the monitor was about $57 \mathrm{~cd} / \mathrm{m}^{2}$ when a neutral gray image was displayed on it.

\section{Free-observing experiment}

Subjects sit on a chair in front of the LCD monitor. The observation distance was $1850 \mathrm{~mm}$ and the view angle of the image at this position was about $29 \mathrm{deg}$. in the horizontal direction. An eye-camera (NAC, EMR-8) was set on the head of a subject to track the eye movement. To prevent any head movement, the chin of the subject was fixed gently to the stand. For the initial 1 second, each image was displayed with a small white cross in the center position. The subject first looked at the cross. After the cross faded away, the subject observed the image freely for about 20 seconds. The 55 wood panel images were switched automatically and the eyemarks of the subject who was observing the images were recorded on videotape. The total observation time was about 30 minutes, including a rest period. After the experiment, the data set of time and coordinates of eyemarks were extracted from the videotape every $1 / 30$ seconds, and summarized to a small data set of eye fixation pauses (steadily observed points). In this study, if the eyemarks stayed in the same position for 0.1 seconds or more, this position was considered to be an eye fixation pause.

\section{Sensory evaluation}

After the above free-observing experiment, sensory evaluation of the same visual stimuli was performed by the semantic differential method.

\section{Results and Discussion}

\section{Distribution map of eye fixation pauses}

A contour map corresponding to the observation intensity was formed from the data of the eye fixation pauses (pausingtime and coordinates). This map reveals where on the image each subject was looking at, and how long he/she watched. As a typical example, distribution maps of three subjects for three images are shown in Fig. 1. In spite of the images being the same, the distribution patterns of the eye fixation pauses are very different among the subjects. Interestingly, even if the distribution patterns of a certain image varied very much among the subjects, there was little difference in the result of their sensory evaluation.

\section{Characterization of distribution maps}

In this study the authors obtained over 1,000 distribution maps, with 20 subjects observing 55 kinds of wood panel images. We qualitatively compared these maps, and found it possible to classify the subjects based on typical characteristics of the distribution patterns. A huge amount of research in the field of experimental psychology has been performed using the eye-tracker, and the method of observation is variously analyzed based on eye fixation pauses; for instance, the difference of eye movements between a veteran car driver and a beginner has been amply demonstrated (e.g., Yamada and Fukuda, 1993). However, there is not much research that pays attention to the distribution pattern of eye fixation pauses. To the best of the authors' knowledge, no attempt has been made that tries to characterize these patterns.

We proposed the following two indexes to indicate the properties of the distribution patterns quantitatively. 1) The aspect ratio $R_{V H}$ is denoted as:

$$
R_{V H}=V / H
$$

where $V$ and $H$ are vertical and horizontal sizes of the distribution, respectively. 2) The rate of expansion $R_{A^{\prime} A}$ is defined as:

$$
R_{A^{\prime} A}=A^{\prime} / A
$$

where $A$ is an area of the wood panel image and $A^{\prime}$ is an area 


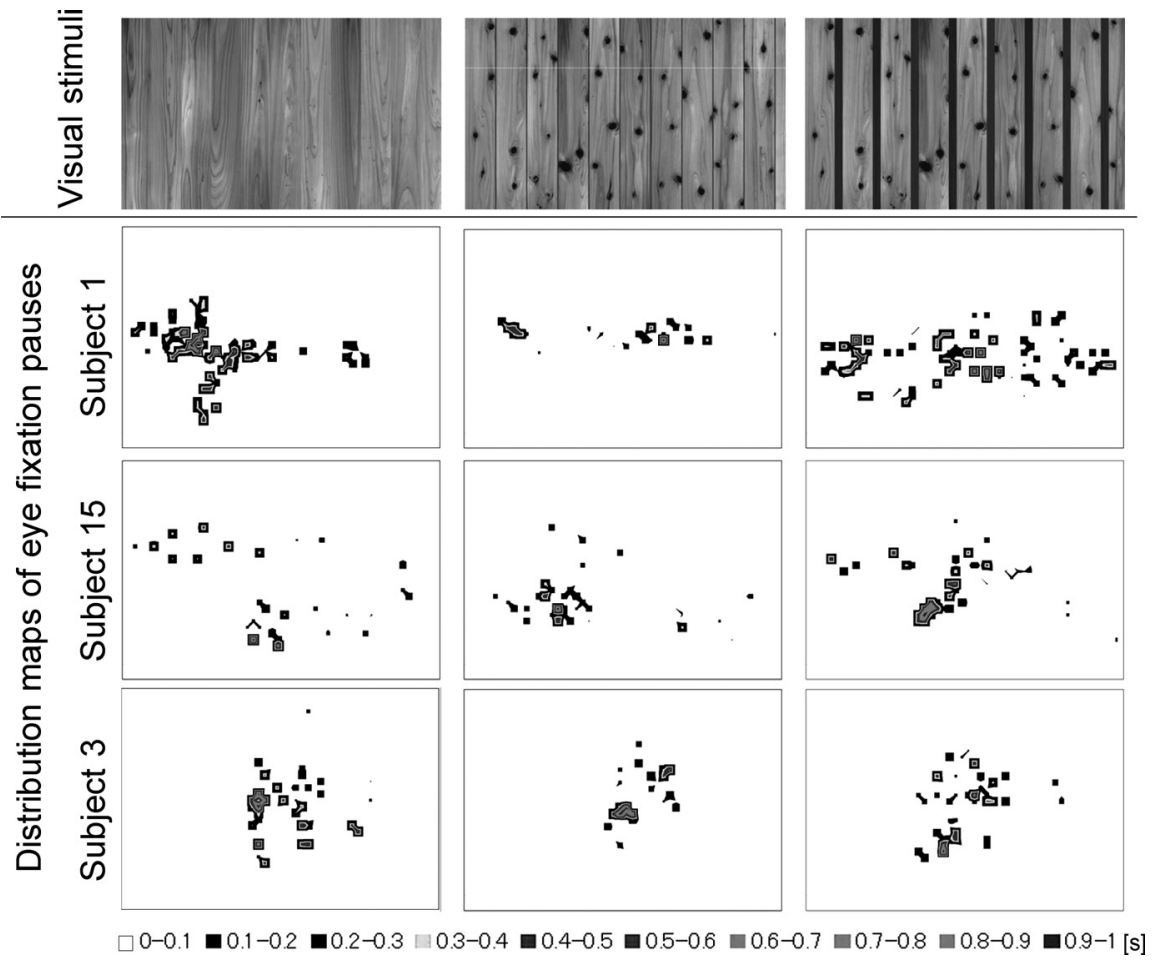

Fig. 1 Examples of visual stimuli and distribution map of eye fixation pauses.

of the distribution. In this study, $A$ is a constant and $A^{\prime}$ is denoted by $A^{\prime}=H V . R_{V H}$ indicates the overall shape of the distribution pattern and $R_{A^{\prime} A}$ corresponds to the coverage of the eye movement on the wood panel image.

The $R_{V H^{-}}$-value and $R_{A^{\prime} A^{\prime}}$-value were calculated using the coordinates-data of eye fixation pauses (Fig. 2), and the relationships between both indexes are summarized in Figure 3. In Figure 3, white dots indicate the observation for the initial 5 seconds, and black dots indicate all observation times (18 seconds). As the $R_{V H}$-value (horizontal axis) grows over 0.6, the shape of the distribution becomes more slender and conversely, as the $R_{V H^{-}}$value becomes less than 0.6 , the shape of the distribution becomes flatter, because the aspect ratio of the wood panel images is about 0.6. On the other hand, an $R_{A^{\prime} A^{-}}$ value (vertical axis) approaching 1.0 means that eye fixation pauses are spreading to the entire image.

In the initial 5 seconds of observation (white dots), white dots are scattered in a similar way to many of the diagrams of Figure 3; dots with big $R_{V H^{-}}$-values and small $R_{A^{\prime} A^{\prime}}$-values, in particular, stand out. This means that many subjects tended to move their eyes more vertically than horizontally in the first stage of the observation. However, in the total 18 seconds of observation (black dots), clear linear relationships between $R_{V H^{-}}$-values and $R_{A^{\prime} A^{\prime}}$-values appeared in some subjects. To evaluate this linearity, the single correlation coefficients $(r)$ between $R_{V H^{-}}$-values and small $R_{A^{\prime} A}$-values were calculated for each subject, and scatter diagrams in Figure 3 were arranged according to $r$-values.

Relatively high linear relationships between $R_{V H}$-values and

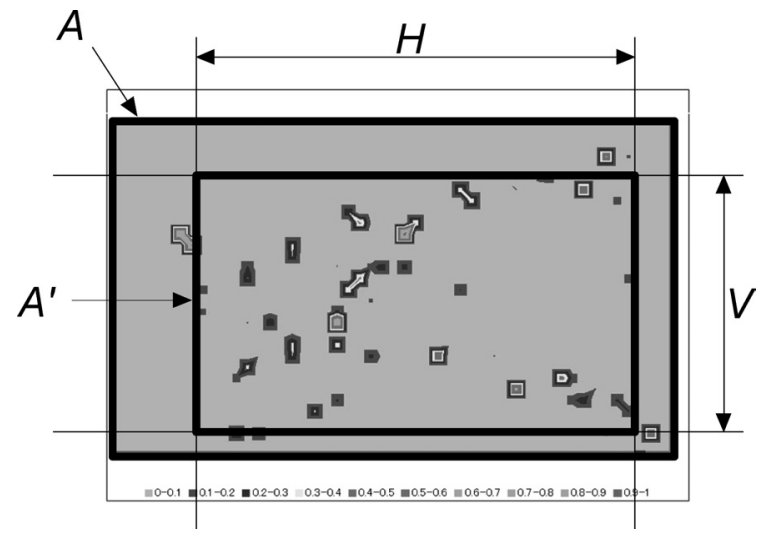

Fig. 2 Schematic illustration for the calculation of two indexes. Note) $H$ : horizontal size of the distribution of eye fixation pauses, $V$ : vertical size of the distribution, $A$ : area of the wood panel image, $A^{\prime}$ : area of the distribution. $A^{\prime}=H V, R_{V H}=V / H, R_{A^{\prime} A}=A^{\prime} / A$. Boundaries of area $A^{\prime}$ were determined based on the averaged coordinates of eye fixation pauses that existed in a periphery of the image. In the case of 18 seconds of observation, 4 points were used for each side. Because of this, the boundaries of $A^{\prime}$ do not match most peripheral eye fixation pauses on the image.

$R_{A^{\prime} A^{\prime}}$-values appear in the diagrams arranged in the left column of Figure 3. The meaning of this high linearity is formulated as follows: The slope of this linear relationship $k$ is:

$$
k=R_{A^{\prime} A} / R_{V H}
$$

where $k$ is a constant of each subject. Inserting eqs. (1) and (2) into eq. (3) gives: 

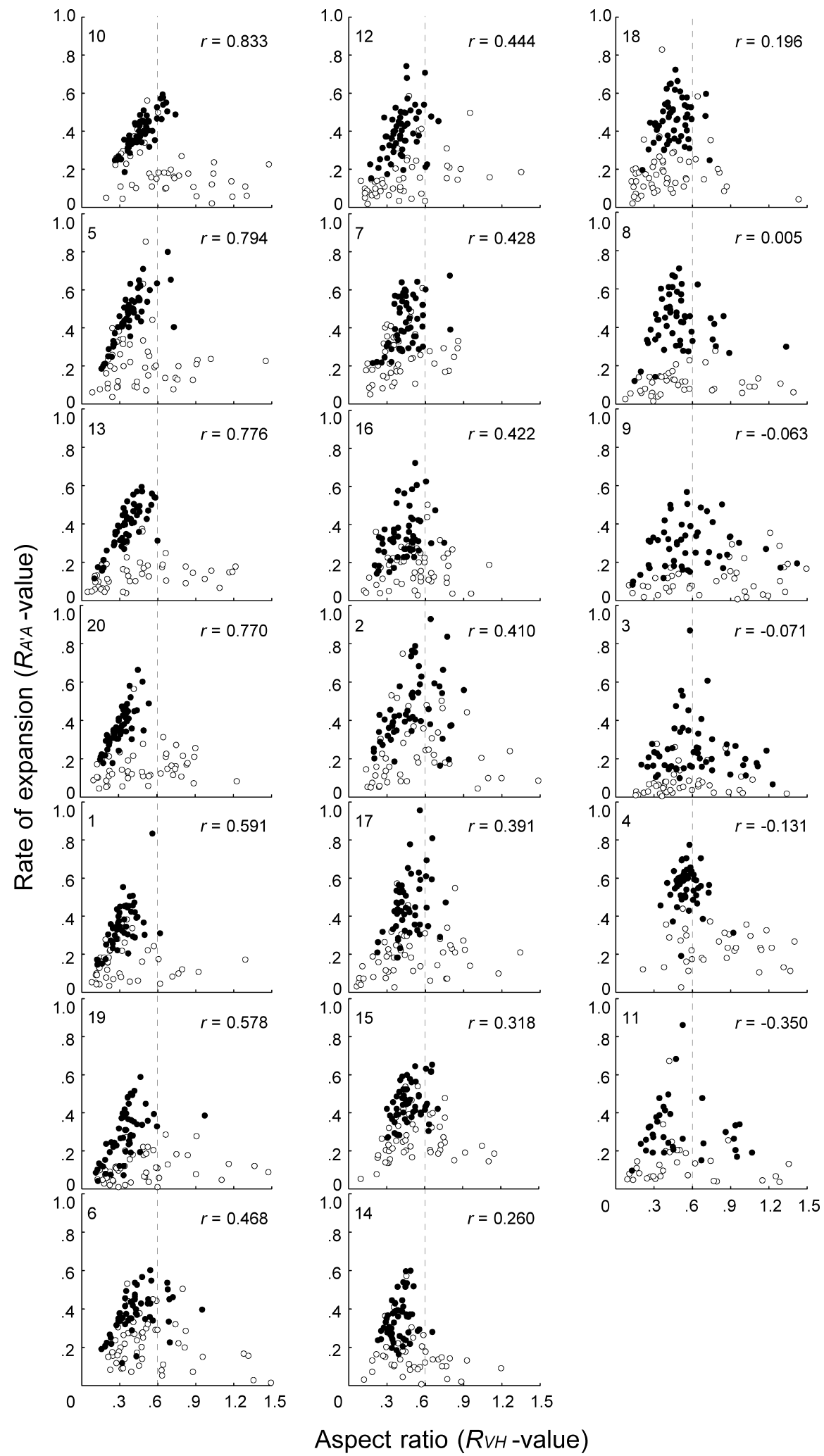

Fig. 3 Relationships between the aspect ratio and the rate of expansion. Note) White dot: initial 5 seconds of observation, black dot: total 18 seconds of observation. The figure at the upper left of each diagram is the subject number. $r$ : single correlation coefficient between $R_{V H^{-v a l u e}}$ and $R_{A^{\prime}}$-value in total 18 seconds of observation (black dots). Dotted line: aspect ratio of the wood panel images. 


$$
k=\left(A^{\prime} / A\right)(H / V)=(H V / A)(H / V)=H^{2} / A .
$$

Equation (4) explains that if $H$ is a constant, $k$ is kept as a constant, because $A$ is a fixed value (the area of the wood panel image). In other words, the subjects who expressed linear relationships in their $R_{V H^{-}}-R_{A^{\prime} A}$ diagrams tended to observe the wood panel images without changing the observation width in any observation.

On the other hand, eq. (4) also represents that the subjects who showed a very weak linearity in $R_{V H}-R_{A^{\prime} A}$ relations changed the observation width according to the wood panel image. Their diagrams are brought together in the right column of Figure 3. Instead of keeping the same observation width, this group tended to maintain the same observation area in any wood panel image, because their ranges of $R_{A^{\prime}}$-values have no big difference from those of the left column group in Figure 3.

The subjects observed the wood panel images freely without any task in the experiment, therefore each subject must have observed these visual stimuli in his/her own way with the least stress and the maximum gain. The visual information collecting styles must have varied widely among them. However, Figure 3 presents the possibility that these styles can be classified into a number of types using proper numerical indexes: subjects who try to keep a fixed observation width (the left column of Fig. 3), subjects who try to maintain a fixed observation area (right column), and an intermediate type (center column). Though it is very attractive to be able to classify the distribution patterns of the eye fixation pauses as observation styles, it should be noted that such a characterization in this study was performed by using only the visual stimuli of wood panel images, limited and specialized as they are. To overcome this limitation, it is necessary to confirm whether a similar classification is possible to more general visual stimuli, such as natural scenes and paintings.

\section{Conclusion}

The distribution of eye fixation pauses obtained from 20 subjects who observed 55 kinds of wood panel images freely were investigated in detail. Even if subjects observed the same images, their distribution patterns were very different. The property of the distribution was characterized using two numerical indexes, the aspect ratio and the rate of expansion. The observation styles of the subjects were classified into three types based on the relationships between the two indexes: observation width constant type, observation area constant type, and an intermediate type. As a future issue, it is necessary to confirm whether a similar classification is possible using other visual stimuli, such as natural scenes and paintings. The method and technique of this study should be combined with simultaneous measurements of physiological responses.

Acknowledgement This work was partly supported by a Grant-in-Aid for Scientific Research (No.15207021) from the Japan Society for the Promotion of Science.

\section{References}

Broman NO. (1995) Attitudes toward Scots Pine Wood Surfaces: A multivariate Approach. Mokuzai Gakkaishi, 41 (11): 994-1005

Broman NO. (1996) Two Methods for Measuring People's Preferences for Scots Pine Wood Surfaces: A Comparative Multivariate Analysis. Mokuzai Gakkaishi, 42(2): 130-139

Broman NO. (2001) Aesthetic properties in knotty wood surfaces and their connection with people's preferences. J Wood Sci 47(3): 192-198

Masuda M, Nakamura M (1987) Influence of Knots on Psychological Images of Panels. Bull Kyoto Univ Forests 59: 273-282 [In Japanese with English Abstract]

Nakamura M, Masuda M, Inagaki M (1993) Influence of Knots and Grooves on Psychological Images of Wood WallPanels. Mokuzai Gakkaishi 39(2): 152-160 [In Japanese with English Abstract]

Sakuragawa S, Miyazaki Y, Kaneko T, Makita T (2005) Influence of Wood Wall Panels on Physiological and Psychological Responses. J Wood Sci 51(2): 136-140

Yamada M, Fukuda T (1993): Eye movements and cognition of pictorial display. In Osaka R, Nakamizo Y, Koga K, eds. Experimental psychology of eye movement. The University of Nagoya Press, Nagoya, 199-217 [In Japanese]

This article was presented at the 8th International Congress of Physiological Anthropology, 2006 (ICPA 2006), in Kamakura, Japan.

Received: September 29, 2006

Accepted: December 5, 2006

Correspondence to: Masashi Nakamura, Division of Forest and Biomaterials Science, Graduate School of Agriculture, Kyoto University, Kitashirakawa, Oiwake-cho, Sakyo-ku, Kyoto 6068502, Japan

Phone: +81-75-753-6237

Fax: +81-75-753-6300

e-mail: nakamasa@kais.kyoto-u.ac.jp 